\title{
Studies on the Fluctuation Characteristics of money- supply in China Based on empirical mode decomposition
}

\author{
Lei-Hong $^{1}$ Huang-JunYing ${ }^{2}$ \\ ${ }^{1}$ School of Business Administration Jimei University \\ ${ }^{2}$ School of Business Administration Jimei University
}

\begin{abstract}
In this paper, the empirical mode decomposition (EMD) method of the wavelet transformation is introduced into the processing to economic time series data, and explored the evolutionary law and development trends of money supply in different time scales, and analyzed the trends of each component and its influence factors. Results show that, EMD can more accurately extract the components with the different cycle of volatility in the time series of money supply, so there should be a broad application prospects in analysis and modeling for macroeconomic data.
\end{abstract}

Keywords: EMD; money supply; multiple time scale; fluctuation characteristics

\section{Introduction}

Money supply, also known as money stock, is the total amount of money in a society at a given point bearing the circulation and payment means. According to the differences in statistical standards, the money supply is divided into $^{M_{0}}$ (cash), $\boldsymbol{M}_{1}\left(\boldsymbol{M}_{\mathrm{o}}+\right.$ demand deposits of commercial banks), $M_{2}\left(M_{1}+\right.$ commercial bank time deposits), $M_{3}\left(M_{2}+\right.$ deposits of other financial institutions), $M_{4}\left(M_{3}+\right.$ large negotiable certificates of deposit CDs), $\boldsymbol{M}_{5}\left(\boldsymbol{M}_{4}+\right.$ short-term government bonds and savings bonds), ${ }^{M_{6}}\left({ }^{M_{5}}+\right.$ short-term commercial paper). While the central bank paid attention to the monitoring and regulation for ${ }^{M_{1}}, \boldsymbol{M}_{2}, \boldsymbol{M}_{3}$ in most countries. When Friedman (1968) investigated the relationship between the money supply and the inflation in the United States during the period 1867 to 1960 , He taking into accounts the impact of time deposits, and adopted $\boldsymbol{M}_{2}$ as the standard to measure the money supply, there is some rationality. In examining impact of money supply on the economic growth, economists widely adopted $\boldsymbol{M}_{1}$ or ${ }^{M_{2}}$ as research object. But which is the better standard; economists have not reached a consensus. Based on general view, we taken $\boldsymbol{M}_{2}$ as object to study[1].With the transition of the economy system from a planned-oriented to market-oriented in China, the money supply was not the exogenous variable decided by the will and policy of the central bank yet, but it is random endogenous variable interacted by the central bank, and a large number of financial institutions, and public, and many economic factors[2]. Only it meets the requirements of economic growth then the money supply can promote sustainable, rapid and healthy economic development. The size of the money supply is an important factor that affects the normal operation of the national economy. Money supply plays a significant supporting role in economic and social development. Under the context of International financial and economic crisis in recent years, studies on the change characteristics of the money supply play a great significance in ensuring the sustainable economic and social development[3].As the money supply is important to the economic growth, the issue of money supply is paid more and more attention and studies on the issue of money supply are gradually deepen. They very early started to research the change characteristic of monetary aggregates overseas[4-5], However, previous studies of domestic scholars focused on exploring the interaction between the money supply and other economic variables[6-8], they used traditional tool to make statistical analysis, and didn't make specific analysis on change characteristic of money supply itself. In this paper, we took EMD as an analytical tool, to explore the cycle characteristics of money supply from the multi-time scale in China, and given references to understand the variation of money supply in China.

In recent years, wavelet transform theory achieved fruitful in multi-time scale analysis of engineering variable. Wavelet transform, known as the mathematical microscope, due to a good ability to multi-resolution analysis in both time domain and frequency domain. But the wavelet transform is essentially a Fourier transformation with adjustable window, and the signal in the wavelet window must be stable, therefore they are not free themselves fundamentally from the limitations of Fourier analysis. Although the wavelet transform get a higher resolution in the frequency domain and time domain at the same time, there are still some restrictions, which often cause a lot of false harmonic wave. And the choice of wavelet basis function has significant influence on wavelet decomposition results[9-10]. Based on local time scale features of signal, Huang etc. proposed and improved empirical mode decomposition (EMD) method, and extracted intrinsic mode function from the original signal [11-12]. This method is actually a smooth processing for signal. It decomposed progressively fluctuations or trends in signals with different scales (frequency), and produces a series of characteristic information of the IMF component containing 
the original signal at different time scales, so each decomposed IMF components has obvious physical background.

The IMF components with the lowest frequency usually represented the trend or mean of original signal. Under the linear framework Hilbert spectrum based on EMD would reach the same performance as wavelet spectrum, while resolution of Hilbert spectrum is much higher than the wavelet spectrum in the frequency domain and time domain. So the results can more accurately reflect physical properties of the signal system. As EMD method has a stronger local performance capabilities than the wavelet method, EMD method is better in dealing with non-linear or non-stationary signals. And stock market data, currency data, exchange rate data and other economic time series data are a typical nonlinear and non stationary time series data, so EMD method is more suitable for analysis and processing these data.

In order to study change laws of money supply better and provide scientific guidance for economic operation and management, in this paper we attempt to use the improved wavelet transform-EMD method for the wavelet transform shortcomings, which gets a significant conclusion by precise decomposition of money supply time series of the multiresolution from 1950 to 2010and discussion of fluctuation component in the characteristics, trends and factors.

\section{The Basic Theory and method of EMD}

If the number of maximum or minimum of data sequence to be decomposed is 2 more than the number of cross-zero over the previous (or next zero crossing) or much more, the data sequence needs smooth processing. Empirical mode decomposition method (EMD) is a new processing method of non-stationary data series, original data can be said by intrinsic mode functions IMF components and a mean value or trend of items through smooth treatment. The basic idea of EMD decomposition method is to characterize the original signal into a series of time-scale IMF components, making the IMF components are narrowband signal. IMF components must meet the following two conditions: First, in the entire signal length, the number of extreme points must be equal with or at most one more than the number of zerocrossing; Second, at any moment, the point average defined by the maxima on the envelope and by the minimum under the envelope is zero, in other words, the upper and lower envelope of the signal line of symmetry is on the timeline. EMD decomposition, also known as the sifting processing, has two functions: remove overlapping wave and make data more symmetrical waveform.

The specific steps as follow:

(1) Finding all the maxima points of original signals, and fit on upper envelope of the original signal with cubic spline function. Similarly, found out all the minimum points of original signals and fit the lower envelope.

(2) Calculating the mean of the upper and lower envelope, denoted by $\mu_{1}(t)$; a new data series that removed the low frequency from original signal can be calculated by the following formula: $q_{1}(t)=m(t)-\mu_{1}(t)$ (1).

(3) Theoretically, $q_{1}(t)$ is a IMF of original signals, but generally $q_{1}(t)$ does not meet the IMF conditions, so we repeat last process $\mathrm{k}$ times for $q_{1}(t)$ and get: $q_{1 k}(t)=q_{1(k-1)}(t)-\mu_{1 k}(t)$ (2).Here $q_{1 k}(t)$ is screened from the $\mathrm{k}$ time, and $q_{1(k-1)}$ is screened from the k-1 time. Until $q_{1 k}(t)$ meets the definition of IMF requirements and the resulting mean value tends to zero, or $q_{1 k}(t)$ meets the following restrictions standard deviation $\mathrm{D}$.

Standard deviation $D=\sum_{k=1}^{T} \frac{\left|q_{1(k-1)}(t)-q_{1 k}(t)\right|^{2}}{q_{1(k-1)}{ }^{2}(t)}$ (3) . Here $\mathrm{T}$ is the length of time series data.

Then we can make use of standard deviation to determine whether screened results are IMF components. Generally, when $\mathrm{D}$ meets $0.2<\mathrm{D}<0.3$, we finished EMD decomposition. There are two purposes that D took the value. On the one hand, make $q_{k}(t)$ even get close to IMF enough; on the other hand, avoid excessively decomposition of the IMF so that selected components preserve amplitude modulation information of original signal.

When $q_{k}(t)$ meets requirements of $\mathrm{D}$, we got the first component $d_{1}(t)$ of IMF ,Let $d_{1}(t)=q_{1 k}(t)$, we got the first IMF component of signal $m(t)$, which represented component with the highest frequency in signal $m(t)$.

(4) Separating $d_{1}(t)$ from $m(t)$, and got a difference signal that the highest frequency components $g_{1}(t)$ removed. That is $g_{1}(t)=m(t)-d_{1}(t)$.Consider $g_{1}(t)$ as original signals, repeat steps(1), (2)and(3) and obtained $d_{2}(t)$ as the second IMF component. Repeated $\mathrm{n}$ times and obtained $\mathrm{n}$ IMF components,

is $\quad d_{1}(t), d_{2}(t), \cdots, d_{n}(t) \quad, \quad g_{1}(t)-d_{2}(t)=g_{2}(t) \quad$, $g_{2}(t)-d_{3}(t)=g_{3}(t), \ldots, g_{n-1}(t)-d_{n}(t)=g_{n}(t)$ (4)

When $d_{n}(t)$ or $g_{n}(t)$ satisfies the given termination conditions (The information of the residual signal $g_{n}(t)$ is less significant to content or a monotone function), decomposition stop and end loop. according (3) and (4), we got: $m(t)=\sum_{i=1}^{n} d_{i}(t)+g_{n}(t)$

Where, $g_{n}(t)$ known as residual function, represented the average trend of signal data. Each IMF component such as $d_{1}(t), d_{2}(t), \cdots, d_{n}(t)$ contains respectively characteristics components of signals at different time scales, which is from small to large. Thus, each component also includes a corresponding section of different frequency components from high to low. Each frequency band contains different frequency components and changes with the signal itself. The specific form can be expressed as follow[13]:

$m(t)=\sum_{j=1}^{n} C_{j}(t)+r_{n}(t)$ 
$C_{j}(t)=A_{j} \sin \left(2 \pi / T_{j}+\phi_{j}\right)+x_{j}$

$r_{n}(t)=x_{r} e^{r t}$

By EMD decomposition principle the result got as follow: $m(t)=\sum_{j=1}^{n}\left[A_{j} \sin \left(2 \pi / T_{j}+\phi_{j}\right)+x_{j}\right]+x_{r} e^{r t}$

There $\mathrm{m}$ is the annual money supply; $\mathrm{t}$ is time; $\mathrm{r}$ is yearly growth rate of money supply in the period; $T_{j}$ is the cycle. EMD method screens by the local characteristics of the signal from the smallest time scale to obtain the shortest cycle of the local IMF components. Then, through layers of screening, we can get IMF components whose local cycle length gradually increases. This process is also reflected multi-resolution analysis of the filtering process. Each IMF component has a clear physical meaning and contains a certain range of characteristic scale, so we can use this feature to filter the signal[14].

\section{Data Processing And Results Analysis}

\section{1. data resources}

Data sources: Zhao-JinWen: An Empirical Study of China's monetary policy and economic growth. Peking UniversityPress,2007.Li-BianHua:The quality Analysis on Chinese economic growth, China Financial and Economic Publishing House, 2008. Yang Qidi, Tu Zhishou. The changes of money supply. Wang Tongsan, Zhang Shouyi, Wang Chongju in chief Editor. Quantitative economics in 21 century (the second volume).Chongqing: Chongqing press,2002.China economy network (Education

Edi-

tion)http://210.34.157.47/index/showdoc.asp?blockcode=D Bjjnj_gdp\&filename=200707311487.http//www.pbc.gov.cn .

\subsection{EMD decomposition of the money supply}

As the length of the actual data is limited, data beginning and terminals are vulnerable to the impact of border effects. While the time series data analyzed with wavelet transform, that the most important is the trend of the end point, so it is necessary to extend data that eliminated boundary effects. There are common approaches such as zero boundary method, symmetric extension method, similarly extension method and the trend extended boundary method. Here we selected symmetric extension method to process data, and solves overshoot and undershoot phenomenon. ${ }^{[13]}$ Specific method as follows: Supposed the original data sequence

: $f(1), f(2), \cdots, f(n)$;n points forward extension: $f(-i)=f(i+1),(i=0,1, \cdots, n-1) ; n$ points backward extension: $f(i+n)=f(n+1-i),(i=1, \cdots, n)$ Got a time sequence that the length is 3 times of original time series, that is as wavelet transform data. As wavelet transform completed, retaining only the wavelet coefficients of the original time period, nature of the boundary does not appeared distortions.
Decomposed the above data with multiple time scale we use EMD method, let D value is 0.25 , and deal with the decomposition effect of end with polynomial fitting algorithm. Results shown in table 1.Table1 are the variance contribution of IMF component and trend component Res.

Table 1. Variance contributions of IMF components

\begin{tabular}{|l|l|l|l|r|}
\hline & IMF1 & IMF2 & IMF3 & RES \\
\hline $\begin{array}{l}\text { Quasi-periodic } \\
\text { (years) }\end{array}$ & $8-13$ & $\begin{array}{l}16- \\
25\end{array}$ & 30 & $\infty$ \\
\hline $\begin{array}{l}\text { Variance con- } \\
\text { tribution }\end{array}$ & 3.7 & 8 & 15.2 & 73.1 \\
\hline
\end{tabular}

\subsection{Conclusions}

(1) Time series data can be decomposed into three different cycles of IMF components and one trend component Res, reflecting money supply to control changes of economic system variables with complex time scales.

(2) The IMF1 is a wave with the maximum amplitude, highest frequency and shortest wavelength. Following IMF component decreased in amplitude, gradually decreased in the frequency, and gradually increased in the wavelength in order.

(3) IMF1 component has quasi-cyclic fluctuations of 8-13 years, whose volatility shows a trend of gradual decay from 1950 to 2010.

(4) IMF2 component has quasi-cyclic fluctuations of 16-25 years, whose volatility decrease continuously in the observed period.

(5) IMF3 component has quasi-cyclic fluctuations of 30 years, whose volatility decrease intensely in the observed period.

(6) Res component shows the overall money supply trends .From 1950 to 2010, the overall money supply trend showed a slow increase by $7.03 \%$, while from 1950.1 to 2010 ,increased drastically up to $35.3 \%$. It must be noted that the trend of money supply may belong to a part of a longer cycle (less frequency), but due to limitation of length of observation period and the sample size, this fluctuation cycle, frequency and amplitude are still difficult to detect.

\subsection{Discussions}

Results shown cycle of large-scale fluctuations (30 years quasi-cycle and trend component) controls the money supply changes in the global timing and cycle of short-scale fluctuations (8-13 years quasi-cycle, 16-25 years quasicycle) become more pronounced than large-scale oscillations in the entire sequence of cyclical fluctuations. Discount and deposit reserve currency is the main input variables and output variables in the money supply system and both of them have complex natural connection. For long sequences, fluctuation of discount amount of money is the most important factor in currency circulation changes, while fluctuation of discount amount of money is controlled and influenced by international and domestic macroeconomic situation changes. Quasi-cycles of fluctuation in 
money supply 8 to 13 years, and $16-25$ years are consistent with the cycle of China's macroeconomic development and quasi-cycle international economic changes, while the quasi-cycle of 30 years in money supply fluctuation may be relevant with long-wave cycle of economy increase.

In this paper, multiple time scale analysis of money supply sequence in 1950-2010 using EMD method shows that fluctuation components are respectively quasi-cycles with 8-13 years, 16-25 years and 30 years, and shows the nature of multiple time scale, multi-level nature and complexity of control of money supply changes in the economic system variable. In addition, it provides scientific guidance for operation and management of macroeconomic variables. As a new non-linear and non-stationary signal analysis theory, EMD method has broad application prospects in the economic system variables over time scales of analysis, modeling and forecasting.

\section{References}

[1] N. Refer Chen Jinmei. Theoretically and practically statistical analysis on macro economy. Beijing: Economic science press, 2005, P124 (In Chinese)

[2] Dong Xiaojun. Studies on early warning mechanism of financial risk. Beijing: Economic management press, 2004, P400, P403 (In Chinese)

[3] Yang Qidi, Tu Zhishou. The changes of money supply . Wang Tongsan, Zhang Shouyi, Wang Chongju in chief Editor. Quantities economics in 21 century (the second volume). Chongqing: Chongqing press, 2002, P242P257 (In Chinese)

[4] Richard H. Dai. Chaos economics. Shanghai: Shanghai translation press, 1996, P167-211 (In Chinese)

[5] Chen Ping. Civilized branch and economic chaos and evolutionary economics. Beijing: Economic science press, 2000, P282-294 (In Chinese)
[6] Gao Ling, Li shichun. The empirical analysis on the money supply and CPI in China in recent years . Industry and technology forum, 2008, (11):151-153 (In Chinese)

[7] Liu Jinquan, Zhang Wengang, Liu Zhaobo. Analysis on the short-term fluctuations and long-term equilibrium relationship between money supply growth and inflation rate. China soft science, 2004, (7):39-44 (In Chinese)

[8] Wang Fangqin: empirical analysis on promoting investment growth of fixed asset based on 1978-2007 data in China. China collective economy, 2008, (9):87-88 (In Chinese)

[9] Peng Yuhua. Wavelet transform and its application. Beijing: Science press, 2002. (In Chinese)

[10] Tewfiki AH. On the optimal choice of a wavelet for signal representation. IEEE trans information theory, 1992, 38 (2): 747-765.

[11] Huang NE, Shen Z, Long SR, et a1.The empirical mode decomposition and the Hilbert spectrum for nonlinear and nonstationary time series analysis . Proceedings of the royal society : mathematical, physical and engineering sciences, 1998, 454: 899 - 955.

[12] Huang NE, Shen Z, Long S R. A new view of nonlinear water waves: The Hilbert spectrum. Annual review of fluid mechanics, 1999, 31:417 - 457. 